\title{
Modelagem e previsão de temperaturas médias mensais de Rio Paranaíba/MG utilizando modelos de séries temporais
}

Os modelos de séries temporais são largamente utilizados no estudo de variáveis climatológicas como a precipitação, umidade e temperatura. Neste artigo, aplicouse a metodologia Box \& Jenkins no intuito da obtenção de um modelo estatístico para previsão de valores futuros de temperatura média mensal para a cidade de Rio Paranaíba-MG. Utilizaram-se dados de temperatura média de dezessete anos (janeiro de 2002 a dezembro de 2018) para o ajuste do modelo. Para o período de teste, foram utilizados os dados do ano de 2019. A análise permitiu identificar na série temporal em estudo, a presença dos componentes, tendência e sazonalidade. Diversos modelos do tipo SARIMA (Autorregresivo Integrado e de Médias Móveis Sazonal) foram ajustados. Dentre os modelos, selecionaram-se os que obtiveram os menores valores dos critérios AIC (Akaike Information Criterion), BIC (Bayesian Information Criterion) e EQM (Erro Quadrático Médio). O modelo escolhido foi o modelo SARIMA $(0,1,2)(3,1,0)_{12}$ que traduziu bem a dinâmica temporal da série para fins de previsão. O referido modelo obteve um bom ajuste à série de temperaturas médias observadas, apresentando para um horizonte previsto de dozes meses, um valor de 1,25 para o EQM.

\section{Modeling and forecasting of monthly mean temperatures in Rio Paranaíba/MG using time series models}

\begin{abstract}
Time series models are widely used for climatological variables such as rainfall, humidity and temperature. In this paper, the Box \& Jenkins methodology was applied in order to obtain a statistical model for forecasting future values of monthly mean temperature for the municipality of Rio Paranaíba, MG. Seventeen year data (January 2002 to December 2018) were used to train set. For validation set, data from 2019 were used. The analysis showed trend and seasonality components. Several models of the SARIMA class (Seasonal Autoregressive Moving Average) have been adjusted. Among all tested models $\mathrm{i}$ selected some competing ones that had the lowest AIC (Akaide Information Criterion), BIC (Bayesian Information Criterion) and MSE (Mean Square Error). The chosen model was SARIMA (0,1,2) $(3,1,0)_{12}$, that represented well the dynamic of the series for forecasting purposes. The model obtained the best fit to the series of monthly mean temperatures and was used to make forecasts for the twelve months forward, resulting in MSE = 1,25
\end{abstract}

Keywords: ARIMA; Forecasting model; Seasonality.

Topic: Meteorologia, Climatologia e Mudanças Climáticas

Reviewed anonymously in the process of blind peer.
Received: 02/10/2020

Approved: $24 / 11 / 2020$

Frederico Carlos Martins de Menezes Filho (it)

Universidade Federal de Viçosa - Campus Rio Paranaíba, Brasil

http://lattes.cnpq.br/6934146250991610

http://orcid.org/0000-0003-4874-0254

frederico.menezes@ufv.br

Referencing this:

MENEZES FILHO, F. C. M.. Modelagem e previsão de temperaturas médias mensais de Rio Paranaíba/MG utilizando modelos de séries temporais. Revista Ibero Americana de Ciências Ambientais, v.11, n.6, p.251-261, 2020. DOI: http://doi.org/10.6008/CBPC21796858.2020.006.0021 


\section{INTRODUÇÃO}

Ao conjunto de observações ordenadas em intervalos de tempo, descritas por uma função matemática (série determinística) ou por distribuições de probabilidade (processo estocástico), cuja principal particularidade é a relação de dependência ou autocorrelação serial entre observações vizinhas, dá-se o nome de série temporal (BARBOSA et al., 2015).

Deste modo, a análise de séries temporais compreende a busca de alguma relação de dependência temporal entre os dados, identificando o mecanismo gerador da série com o objetivo de extrair periodicidades relevantes nos registros; descrever o seu comportamento e realizar previsões (BAYER et al., 2010). Dentre os modelos estocásticos mais utilizados na análise de séries temporais, destacam-se os modelos ARIMA (Auto Regressive Integrated Moving Average) e suas variações (AR, MA, ARMA, SARIMA, SARIMAX) propostos por Box \& Jenkins (BOX et al., 2016).

Conforme explicitado por Silva et al. (2008), a análise de séries temporais aplicada a fatores climatológicos atrai especial interesse já que tais fatores exercem papel significativo ao sucesso ou fracasso de inúmeros empreendimentos. Os mesmos autores ainda complementam da importância de previsões climáticas sobre o melhor gerenciamento em várias áreas como a agricultura, recursos hídricos e atividade pesqueira. Neste enfoque, modelos estatísticos de previsão utilizando variáveis climatológicas vêm sendo amplamente aplicados em diferentes regiões.

Silva et al. (2008) analisaram a temperatura média mensal na cidade de Uberlândia, MG, utilizandose o modelo de série temporal do tipo SARIMA. Chechi et al. (2012) avaliaram as previsões de temperaturas mínimas e máximas mensais médias da cidade de Erechim, RS verificando a maior acurácia na previsão dos modelos do tipo SARIMA em comparação aos modelos de alisamento exponencial (Holt-Winters). Barbosa et al. (2015), utilizando-se a metodologia Box \& Jenkins, obtiveram o modelo de previsão SARIMA para previsão de temperatura média mensal para a cidade de Bauru, SP, com valores previstos bem próximos dos observados. Pereira et al. (2015), analisando-se séries históricas de precipitação pluvial e temperatura média do ar para a cidade de Areia, PB, obtiveram resultados satisfatórios de previsão utilizando-se de modelos SARIMA. Werner et al. (2018) compararam cinco modelos e suas combinações (suavização exponencial, SARIMA e ARFIMA) para a previsão da umidade relativa do ar diária de Porto Alegre, RS, concluindo que a combinação entre o método de regressão e o modelo ARFIMA proporcionou maiores medidas de acurácia para os dados do estudo.

Verifica-se, portanto, a larga utilização dos modelos estocásticos na previsão de variáveis climáticas em distintas regiões do país. Todavia, na região do Alto Paranaíba-MG, em específico, na cidade de Rio Paranaíba-MG, o estudo do comportamento das variáveis climatológicas é ainda incipiente apesar de ser uma região de grande importância agrícola para o Estado de Minas Gerais.

Deste modo, o presente estudo, objetivou realizar a análise, modelagem e previsões da série temporal de temperaturas médias mensais na cidade de Rio Paranaíba-MG, considerando modelos estocásticos da classe ARIMA, conhecidos como modelos SARIMA. 


\section{METODOLOGIA}

\section{Área de Estudo}

O município de Rio Paranaíba-MG se localiza na latitude de $19^{\circ} 11^{\prime} 39^{\prime \prime} \mathrm{S}$, longitude $46^{\circ} 14^{\prime} 37^{\prime \prime} \mathrm{W}$. Com uma área de aproximadamente $1.353 \mathrm{~km}^{2}$ e pertencente à região do Alto Paranaíba-MG, possui uma população estimada para o ano de 2019 de 12.316 habitantes; sendo a agropecuária sua principal atividade econômica (IBGE, 2019). Segundo a classificação de Köppen-Geiger, o clima da região é do tipo Cwa, clima quente e temperado com menor pluviosidade no inverno em comparação ao verão.

\section{Dados}

Os dados de temperatura avaliados foram obtidos do Sistema de Monitoramento Meteorológico Cooxupé (SISMET-Cooxupé) ${ }^{1}$, referente à estação climatológica situada na cidade de Rio Paranaíba-MG, coordenadas latitude $19^{\circ} 11^{\prime} 43^{\prime \prime} \mathrm{S}$, longitude $46^{\circ} 14^{\prime} 7^{\prime \prime} \mathrm{W}$.

O período da série analisada compreendeu as médias mensais de janeiro de 2002 a dezembro de 2019, totalizando uma série com 216 observações. Os valores relativos ao período de 2002 a 2018 foram utilizados para o ajuste do modelo. Já os dados referentes a 2019 foram selecionados para avaliação das previsões.

\section{Modelo de previsão SARIMA}

Os modelos de previsão do tipo ARIMA (Autoregressive Integrated Moving Average) combinam processos autorregressivos (AR) e de médias móveis (MA), sendo, dentre os modelos tradicionais de previsão existentes, os de abordagem mais avançada e robusta (KRISPIN, 2019).

Em estudos de séries temporais que envolvam variáveis ambientais, observa-se comumente a sazonalidade destas por um padrão de repetição temporal (SILVA et al., 2008; BAYER et al., 2012; BLEIDORN et al., 2019). Nestes casos, os modelos ARIMA passam a incorporar essa característica, sendo denominados de modelos SARIMA, utilizados nesse estudo. O modelo SARIMA pode ser representado pela Equação 1:

$$
\emptyset(B) \Phi\left(B^{12}\right) \Delta^{d} \Delta_{12}^{D} Z_{t}=\theta(B) \Theta\left(B^{12}\right) \varepsilon_{t}
$$

Em que: $\emptyset(B)=1-\emptyset_{1}(B)-\emptyset_{2}\left(B^{2}\right)-\cdots-\emptyset_{p}\left(B^{p}\right)$ é o operador auto-regressivo de ordem $\mathrm{p}$; $\Phi\left(B^{12}\right)=1-\Phi_{1}\left(B^{12}\right)-\Phi_{2}\left(B^{12}\right)-\cdots-\Phi_{q}\left(B^{12}\right)^{q}$ é o operador de médias móveis de ordem q; $\Phi\left(B^{12}\right)=1-\Phi_{1}\left(B^{12}\right)-\Phi_{2}\left(B^{12}\right)-\cdots-\Phi_{P S}\left(B^{12}\right)^{P S}$ é o operador auto-regressivo sazonal de ordem Ps; $\Theta\left(B^{12}\right)=1-\Theta_{1}\left(B^{12}\right)-\Theta_{2}\left(B^{12}\right)-\cdots-\Theta_{Q S}\left(B^{12}\right)^{Q s}$ é o operador de médias móveis sazonal de ordem Qs; $\Delta^{d}$ é o operador diferença simples, sendo d o número de diferenças; $\Delta_{12}^{D}$ é o operador diferença sazonal, sendo $\mathrm{D}$ o número de diferenças sazonais; $\varepsilon_{t}$ é o resíduo aleatório no tempo t.

\section{Sazonalidade}

A existência da sazonalidade foi verificada por meio da análise espectral e pelo teste $G$ de Fisher, admitindo-se 0,05 de significância $(\alpha=0,05)$. A análise espectral consiste na obtenção do periodograma que

\footnotetext{
${ }^{1}$ http://sismet.cooxupe.com.br:9000/
} 
se refere à decomposição da série temporal em uma série de Fourier, no domínio de frequências (MORETTIN et al., 2006). No eixo das ordenadas se localizam as frequências da série (fi) e no eixo das abscissas as respectivas intensidades de cada frequência Ip(fi), definidas pela Equação 2:

$$
\mathrm{I}_{\mathrm{j}}^{(\mathrm{N})}=\frac{1}{2 \pi \mathrm{N}}\left|\sum_{\mathrm{t}=1}^{\mathrm{N}} \mathrm{Z}_{\mathrm{t}} \mathrm{e}^{-\mathrm{i} \lambda \mathrm{t}}\right|^{2}
$$

Em que:

$$
\begin{array}{r}
e^{-i \lambda}=\cos \lambda+i \operatorname{sen} \lambda ; \\
\mathrm{i}=\sqrt{-1} ;
\end{array}
$$

$\mathrm{N}$ é o número de observações da série; $\lambda_{j}=\frac{2 \pi j}{N}$ são as frequências da série de Fourier.

Maiores detalhes podem ser consultados em Morettin et al. (2006).

A periodicidade sazonal pode ser verificada observando a qual frequência fi está associada o maior valor de Ip(fi) e, então, dividir 1 por este valor de frequência. Utiliza-se o teste $\mathrm{G}$ de Fischer para testar a significância do valor obtido, Equação 3. Sendo este superior ao valor de Z, calculado pela Equação 4, concluise que o componente sazonal com periodicidade s=1/fi é significante (BARBOSA et al., 2015).

$$
\begin{aligned}
& G=\frac{\max \left[I_{p}(f i)\right]}{\sum_{i=1}^{\left(\frac{N}{2}\right)} I p(f i)} \\
& Z=1-\left[\frac{\alpha}{\left(\frac{N}{2}\right)}\right]^{\frac{1}{\left(\frac{N}{2}-1\right)}}
\end{aligned}
$$

\section{Modelagem estocástica}

Para o estabelecimento de um modelo da classe SARIMA utilizou-se a metodologia Box \& Jenkins (BOX et al., 2016), definida pelas seguintes etapas: (a) identificação do modelo; (b) estimação dos parâmetros e (c) diagnóstico do modelo.

Dada a grande dificuldade da estimação dos parâmetros por meio das funções de Autocorrelação (ACF) e Autocorrelação Parcial (PACP), a identificação do modelo se deu pela simulação de diversos modelos SARIMA, selecionando-se os seis com os menores valores obtidos para os critérios de seleção, também conhecidos como critérios de informação. Tais critérios utilizados foram o AIC (Akaike Information Criterion) proposto por Akaike (1974) e o de BIC (Bayesian Information Criterion) desenvolvido por Akaike (1978), expressos, respectivamente, pelas Equações 5 e 6:

$$
A I C=-2 \ln [M V]+2 r
$$

Em que: MV é função de máxima verossimilhança e r é o número de parâmetros independentes ajustados.

$$
B I C=-2 \ln [M V]+(r+r \ln N)
$$

Em que: MV é função de máxima verossimilhança; r é o número de parâmetros independentes ajustados e N o número de observações.

Ambas estatísticas buscam o equilíbrio entre modelos parcimoniosos que privilegiam o menor uso de parâmetros quanto possível, e modelos que sejam simples e negligenciem efeitos que apresentem efeitos importantes (CHATFIELD et al., 2019). O melhor modelo será aquele que apresentar menores valores para AIC e BIC.

Deste modo, seis modelos concorrentes foram ajustados e, a partir dos valores dos critérios citados, escolheu-se o modelo mais adequado para realizar previsões. O diagnóstico do modelo selecionado para a 
previsão compreendeu em verificar se os resíduos possuíam características de ruído branco, ou seja, possuindo média nula e variância constante. Para avaliar a presença ou não de autocorrelação serial, dispôsse do teste de Ljung-Box (LUUNG et al., 1978). De modo complementar também fora realizado o teste de normalidade de resíduos por meio do tradicional teste Shapiro-Wilk (SHAPIRO et al., 1965).

Após estas etapas, realizou-se a previsão dos valores de temperatura média mensal para o horizonte de 12 meses, correspondendo aos meses do ano de 2019. A escolha do modelo de previsão, conjuntamente à análise dos valores de AIC e BIC, baseou-se na estatística do Erro Quadrático Médio (EQM) obtido pela Equação 7:

$$
\mathrm{EQM}=\frac{1}{12} \sum_{t=1}^{t=12} e a^{2}
$$

Em que ea é o erro aleatório estimado para cada mês no intervalo de previsão. Todas as análises desenvolvidas nesse trabalho foram realizadas por meio do software livre R (R CORE TEAM, 2017), com a utilização de pacotes específicos para análise de séries temporais, dentre eles o forecast e o TSstudio.

\section{RESULTADOS E DISCUSSÃO}

\section{Análise descritiva dos dados}

Na tabela 1 apresentam-se algumas medidas descritivas da variável temperatura, correspondente à série de médias mensais referentes ao período de janeiro de 2002 a dezembro de 2018 na cidade de Rio Paranaíba-MG, estação SISMET-COXUPÉ.

Tabela 1: Medidas descritivas da variável temperatura em estudo.

\begin{tabular}{|l|l|}
\hline Medida descritiva & Valor \\
\hline Média $\left({ }^{\circ} \mathrm{C}\right)$ & 21,4 \\
\hline Mediana $\left({ }^{\circ} \mathrm{C}\right)$ & 21,6 \\
\hline Desvio padrão $\left({ }^{\circ} \mathrm{C}\right)$ & 1,9 \\
\hline Coeficiente de variação (\%) & 9,3 \\
\hline Valor máximo $\left({ }^{\circ} \mathrm{C}\right)$ & 26,4 \\
\hline Valor mínimo $\left({ }^{\circ} \mathrm{C}\right)$ & 15,4 \\
\hline Assimetria & $-0,3$ \\
\hline
\end{tabular}

A média das temperaturas mensais do período foi de $21,4{ }^{\circ} \mathrm{C}$, com o desvio padrão de $1,9^{\circ} \mathrm{C}$. Os valores baixos para o desvio padrão e para o coeficiente de variação $(9,3 \%)$ indicam que a média aritmética é representativa para a série em estudo.

A temperatura média mensal máxima no valor de $26,4{ }^{\circ} \mathrm{C}$ foi registrada em outubro de 2015 , enquanto que a mínima no valor de $15,4{ }^{\circ} \mathrm{C}$ ocorreu em junho de 2006. A distribuição da série de temperaturas médias mensais possui assimetria igual a -0,3, indicando histograma com assimetria negativa, corroborado pela mediana superior à média.

Na figura 1 apresenta-se um gráfico de linha com a série temporal mensal de temperaturas médias. Observa-se um padrão de variabilidade intra-anual, com períodos de temperaturas elevadas seguidos por períodos de temperaturas baixas, caracterizando a presença de sazonalidade. 


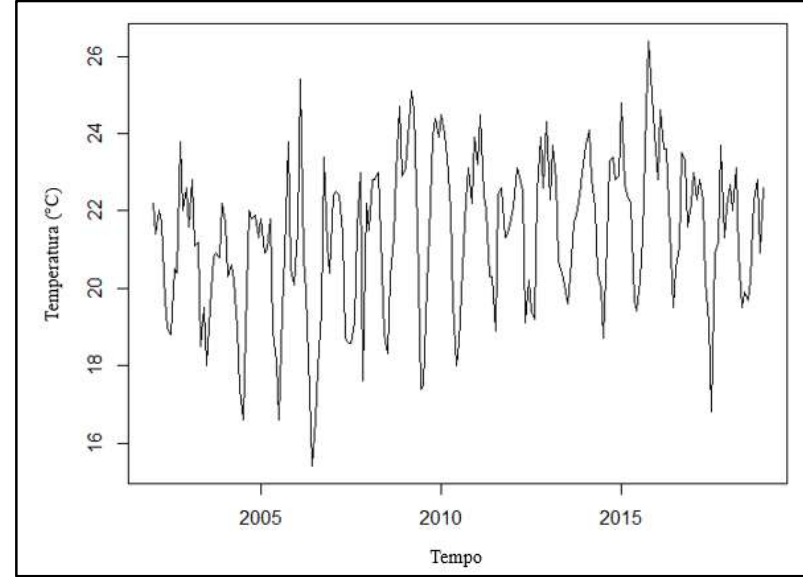

Figura 1: Gráfico da série de temperaturas médias mensais de janeiro de 2002 a dezembro de 2018.

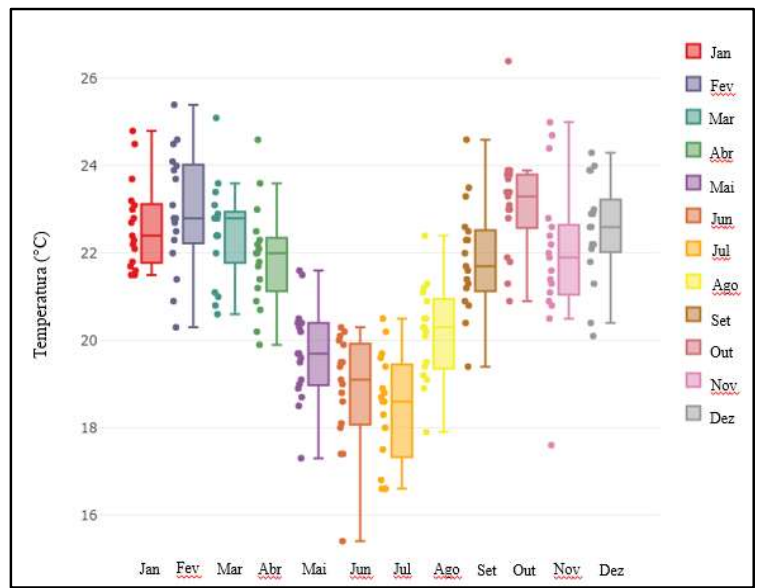

Figura 2: Gráfico da sazonalidade de temperaturas entre os meses de janeiro de 2002 a dezembro de 2018.

Tal fato, pode ser melhor visualizado por meio da figura 2, onde há uma queda da temperatura entre os meses de maio a agosto e o aumento da mesma a partir de setembro, prolongando-se o período de temperaturas mais elevadas até abril.

Na figura 3 apresenta-se a decomposição da série, denotando-se a sazonalidade já visualizada na figura anterior, bem como, a existência de uma tendência positiva a partir de 2006.

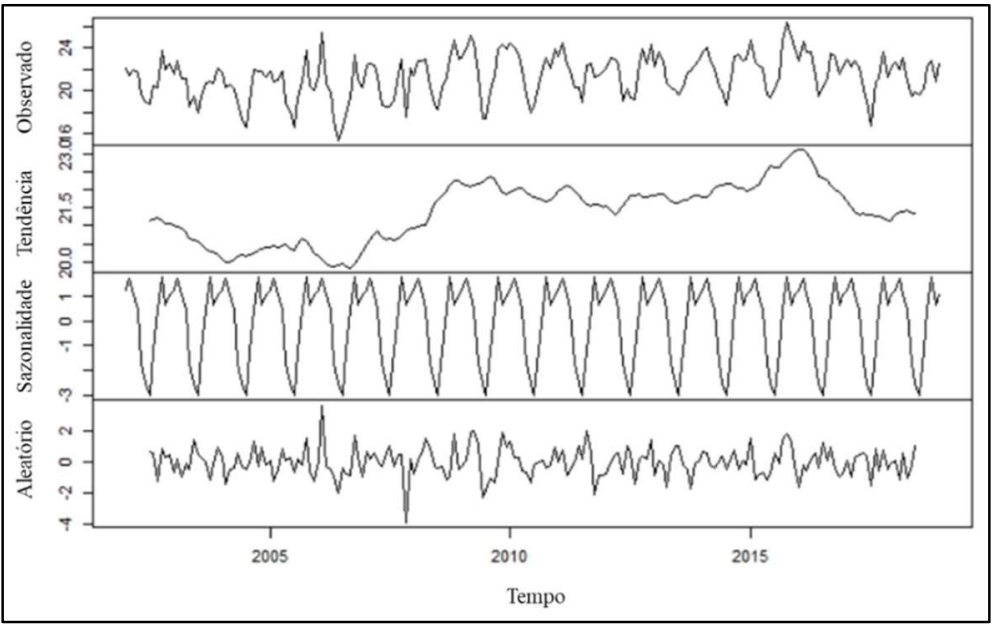

Figura 3: Decomposição da série temporal em componentes de sazonalidade, de tendência e de aleatoriedade.

\section{Modelagem e Previsão}

\section{Identificação do modelo}

A não-estacionariedade da série em relação à média e a variância observada na figura 3 foi confirmada com o uso do teste de Dickey-Fuller (SAID et al., 1984), aceitando-se a hipótese nula de nãoestacionariedade com p-valor inferior a 0,01. Já a significância estatística da tendência foi verificada pelo teste do sinal, também conhecido como Cox Stuart Trend Test (COX et al., 1955). Obteve-se para o referido teste uma tendência positiva com 59 pares positivos contra 43, rejeitando a hipótese nula de não existir tendência, com p-valor de 0,1371. Tais resultados confirmam a decomposição da série apresentada pela figura 3, observando-se tanto a não-estacionariedade quanto a tendência crescente a partir do ano de 2006. A existência de sazonalidade, apresentada visualmente pelas figuras 2 e 3 , foi confirmada através da 
análise espectral e pelo teste $G$ de Fischer $(\alpha=0,05)$. O periodograma apresentado na figura 4 corrobora a sazonalidade com periodicidade de $s=12$ meses, com a maior densidade espectral no valor de 192,14 associada à frequência de 0,083333 . Consoante o teste $G$ de Fisher foram obtidos os valores de $G=0,47$ e $Z$ $=0,073$. Sendo o valor de G superior ao de Z, confirma-se a presença de sazonalidade anual na série de temperatura média mensal.

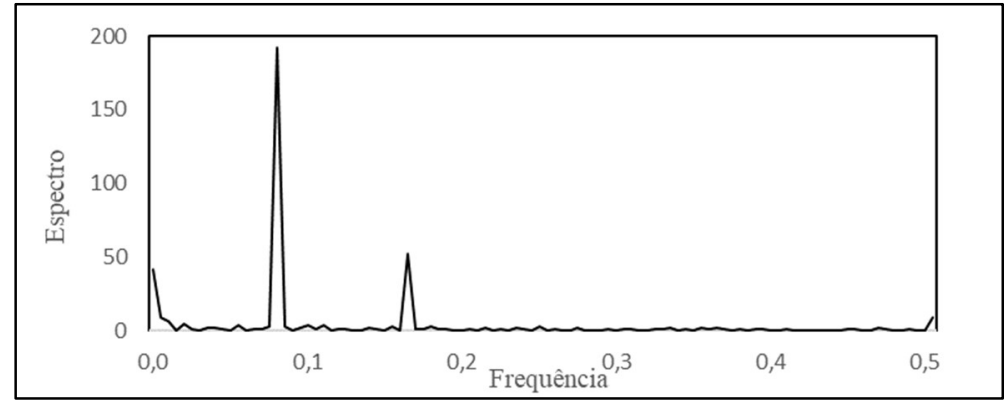

Figura 4: Periodograma da série de temperaturas médias mensais.

Consoante Box et al. (2016), para a identificação dos modelos é essencial observar o comportamento dos correlogramas da série, verificando-se as funções de autocorrelação amostral (FAC) e de autocorrelação amostral (FACP) da série em nível (figura 5) e da série diferenciada de ordem 12, figura 6.

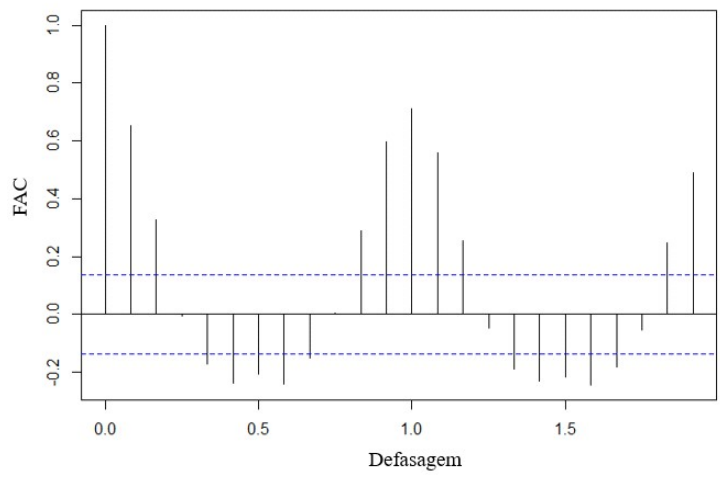

(a)

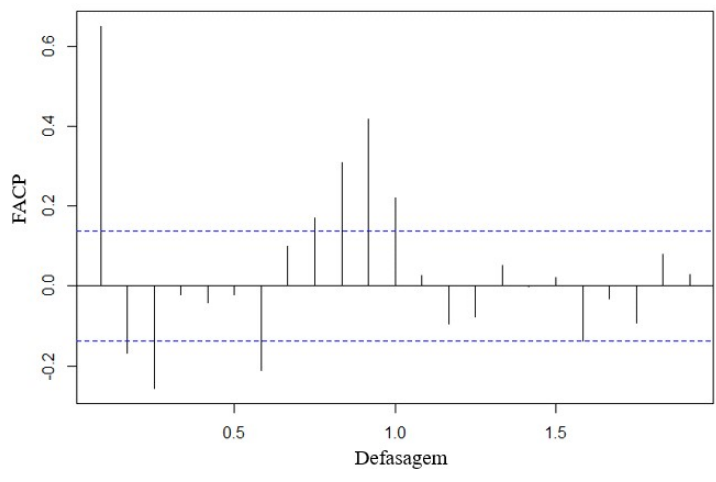

(b)

Figura 5: Função de autocorrelação amostral (a) e função de autocorrelação parcial amostral (b) da série.

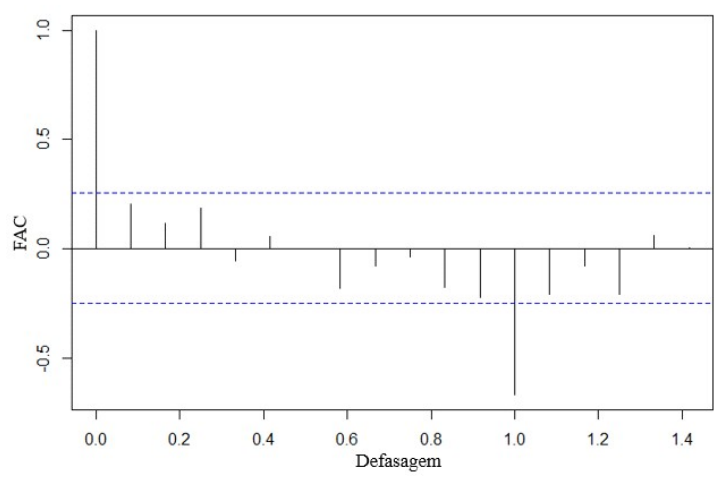

(a)

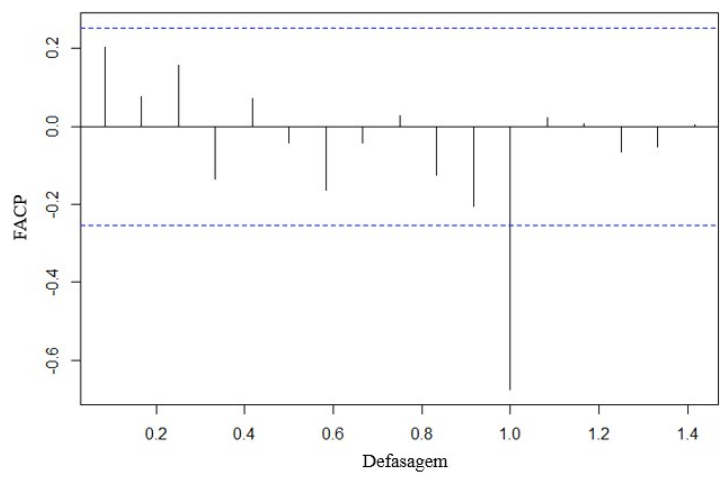

(b)

Figura 6: Função de autocorrelação amostral (a) e função de autocorrelação parcial amostral (b) após aplicação da diferença sazonal.

De acordo com a figura 5, a função de autocorrelação amostral (FAC) indica a sazonalidade da série 
com o correlograma apresentando oscilações nas defasagens múltiplas de 12 com significância diferente de zero, ou seja, fora do intervalo de confiança delimitado pelas linhas tracejadas. Tal fato evidencia correlação serial a ser modelada, além do comportamento senoidal e infinito em extensão da FAC, indicando um processo do tipo auto-regressivo, não estacionário e sazonal.

Na figura 6 após a diferença de ordem 12 aplicada para eliminar a sazonalidade presente na série de temperaturas médias mensais, ainda se nota a defasagem múltipla de 12 significativa tanto na FAC quanto na FACP, indicando a presença de sazonalidade estocástica.

Deste modo, conclui-se que a temperatura média mensal na cidade de Rio Paranaíba possui uma sazonalidade bem definida, como verificada nos correlogramas e na autocorrelação, evidenciando que a mesma pode ser explicada pelas temperaturas dos mesmos meses nos anos anteriores.

Sendo assim, para a determinação do modelo, diversos modelos SARIMA $(p, d, q) \times(P, D, Q)_{s}$ foram ajustados, tais que a ordem auto-regressiva ( $p$ e $P$ ) fosse inferior a 3 e que a ordem das médias móveis ( $q$ e Q) e das diferenças ( $d$ e D) fossem inferiores a 2. Após esta etapa, foram selecionados 6 modelos que apresentaram os menores valores para o AIC, BIC e EQM. Os modelos concorrentes com as medidas de performance calculadas são apresentados na tabela 2 .

Tabela 2: Critérios de escolha do melhor ajuste e melhor modelo de previsão

\begin{tabular}{|l|l|l|l|}
\hline Modelo & AIC & BIC & EQM \\
\hline SARIMA $(0,1,1)(2,1,0)_{12}$ & 631,52 & 644,53 & 2,05 \\
\hline SARIMA $(0,1,1)(3,1,0)_{12}$ & 626,00 & 642,26 & 1,34 \\
\hline SARIMA(0,1,2)(3,1,0) 12 & 623,97 & $\mathbf{6 4 3 , 4 9}$ & $\mathbf{1 , 2 5}$ \\
\hline SARIMA $(1,1,1)(1,1,0)_{12}$ & 657,98 & 670,99 & 1,99 \\
\hline SARIMA $(1,1,2)(2,1,0)_{12}$ & 630,05 & 649,56 & 1,85 \\
\hline SARIMA $(3,1,0)(2,1,0)_{12}$ & 651,47 & 670,98 & 2,53 \\
\hline
\end{tabular}

Todos os modelos selecionados possuem valores para AIC, BIC e EQM muito próximos. Quanto ao ajuste, os modelos SARIMA $(0,1,1)(3,1,0)_{12}$ e SARIMA $(0,1,2)(3,1,0){ }_{12}$ apresentaram os menores valores para os critérios BIC e AIC, respectivamente. Como se observa, o critério BIC penaliza o modelo com o maior número de parâmetros como se verifica para o modelo SARIMA $(0,1,2)(3,1,0)_{12}$. Deste modo, dentre os dois modelos citados, selecionou-se o modelo SARIMA $(0,1,2)(3,1,0)$ de menor EQM, portanto, o mais indicado para fazer previsões.

\section{Diagnóstico do modelo}

Após a definição do modelo e estimativa dos parâmetros, realizou-se a análise de diagnóstico do mesmo com o objetivo de validá-lo para previsões. Na figura 7, apresentam-se os resíduos padronizados que seguem uma distribuição próxima da normal, verificando a homogeneidade dos mesmos, confirmando as características de ruído branco, com o p-valor de 0,54 no teste de Ljung-Box.

Também na mesma figura, apresenta-se o correlograma dos resíduos do modelo onde observa-se a não correlação entre os mesmos em nenhuma defasagem e, por fim, uma interpretação gráfica do teste de Ljung-Box, indicando graficamente para diferentes defasagens que todos os valores estão acima de $5 \%$, não se rejeitando a hipótese nula de que os resíduos são ruído branco. O teste Shapiro-Wilk ( $p$-valor=0,007) 
realizado para o modelo selecionado, indica que os resíduos seguem distribuição normal.

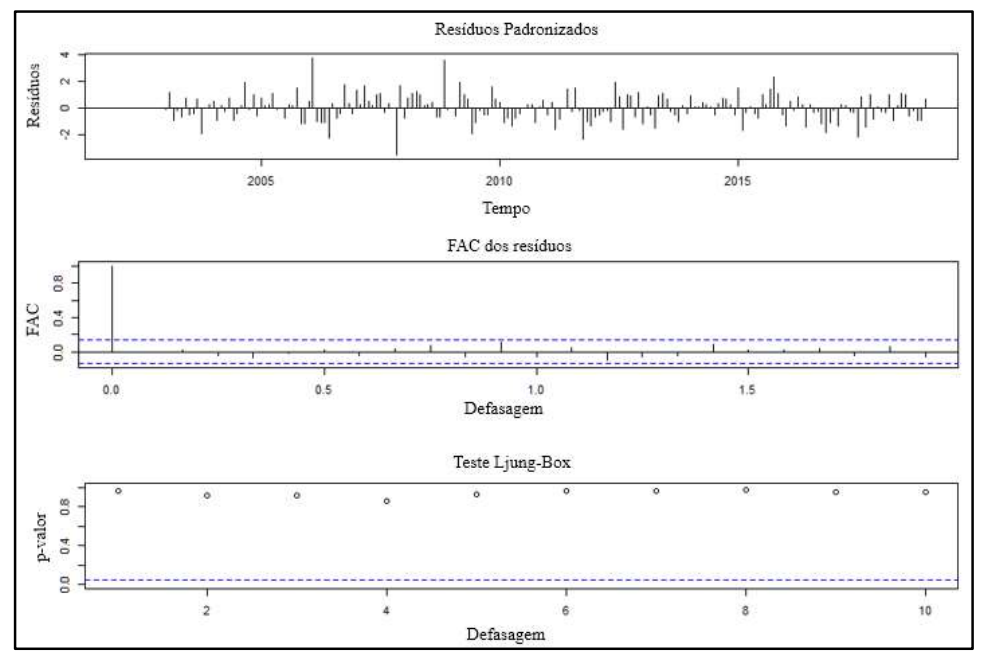

Figura 7: Comprovação das características de ruído branco.

Na tabela 3 apresentam-se as estimativas de máxima verossimilhança dos parâmetros do modelo selecionado, SARIMA $(0,1,2)(3,1,0)_{12}$. Como observado, foram tomadas duas diferenças, uma em relação à tendência e outra em relação à sazonalidade.

Tabela 3: Estimativas dos parâmetros do modelo selecionado

\begin{tabular}{|l|l|}
\hline Modelo & Parâmetros \\
\hline \multirow{4}{*}{ SARIMA $(0,1,2)(3,1,0)_{12}$} & $\Theta_{1}=-0,6928$ \\
\cline { 2 - 2 } & $\Theta_{2}=-0,1397$ \\
\cline { 2 - 2 } & $\Theta_{1}=-0,7010$ \\
\cline { 2 - 2 } & $\Theta_{2}=-0,5252$ \\
\cline { 2 - 2 } & $\Theta_{3}=-0,1945$ \\
\hline
\end{tabular}

A série ajustada com o modelo estimado é apresentada na figura 8. Verifica-se pela ilustração um bom desempenho do modelo, confirmando os resultados da análise residual. Observa-se também valores ajustados próximos aos observados, indicando que o modelo conseguiu traduzir a dinâmica temporal da série histórica de temperaturas médias mensais.

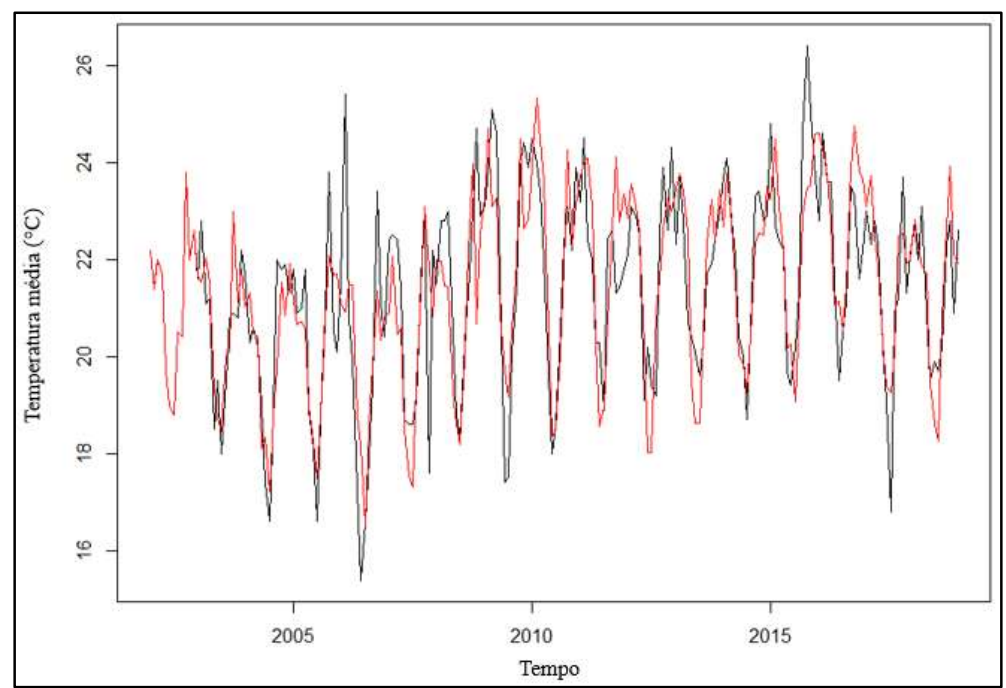

Figura 8: Valores observados (linha escura) e valores previstos (linha vermelha). 


\section{Previsão de temperaturas médias mensais}

Nesta etapa, foram traçadas as previsões de temperaturas médias mensais para um horizonte de dozes meses à frente, correspondente ao ano de 2019. Tais meses não foram utilizados no ajuste do modelo.

Na figura 9 são apresentados os valores observados e os previstos. Os valores observados, obtidos na previsão e os resíduos estão dispostos na tabela 4.

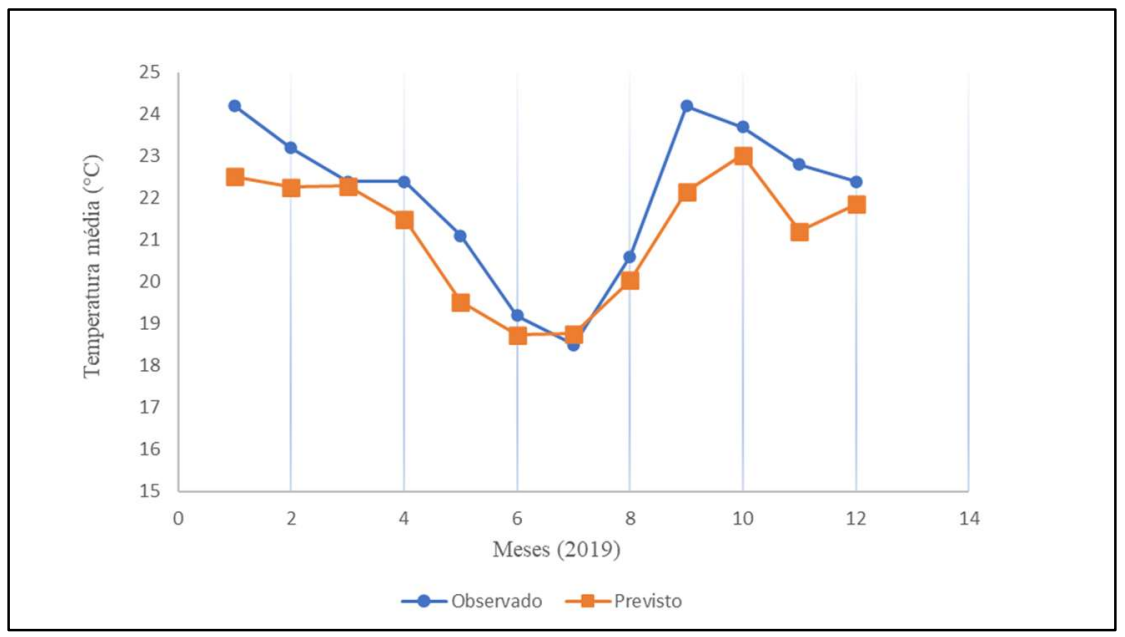

Figura 9: Temperaturas médias observadas e previstas para 2019.

Tabela 4: Valores de temperaturas médias observados e previstos para 2019 com o modelo SARIMA $(0,1,2)(3,1,0)$.

\begin{tabular}{|l|l|l|l|}
\hline Mês - 2019 & $\begin{array}{l}\text { Valores observados } \\
\left({ }^{\circ} \mathbf{C}\right)\end{array}$ & $\begin{array}{l}\text { Valores previstos } \\
\left({ }^{\circ} \mathbf{C}\right)\end{array}$ & Resíduos \\
\hline Janeiro & 24,20 & 22,51 & 1,69 \\
\hline Fevereiro & 23,20 & 22,26 & 0,94 \\
\hline Março & 22,40 & 22,28 & 0,12 \\
\hline Abril & 22,40 & 21,50 & 0,90 \\
\hline Maio & 21,10 & 19,53 & 1,57 \\
\hline Junho & 19,20 & 18,74 & 0,46 \\
\hline Julho & 18,50 & 18,76 & $-0,26$ \\
\hline Agosto & 20,60 & 20,04 & 0,56 \\
\hline Setembro & 24,20 & 22,16 & 2,04 \\
\hline Outubro & 23,70 & 23,03 & 0,67 \\
\hline Novembro & 22,80 & 21,21 & 1,59 \\
\hline Dezembro & 22,40 & 21,86 & 0,54 \\
\hline
\end{tabular}

\section{CONCLUSÕES}

Neste estudo, realizou-se o ajuste de um modelo estocástico da classe ARIMA para uma série de dados de temperaturas médias mensais da cidade de Rio Paranaíba-MG. Para tal, empregou-se a metodologia Box \& Jenkins para previsões e estimativas de temperatura média para o horizonte de doze meses referente ao ano de 2019.

Dentre os seis modelos propostos da classe SARIMA, o modelo que obteve o melhor ajuste aos dados foi o SARIMA $(0,1,2)(3,1,0)_{12}$, apresentando menor Erro Quadrático Médio, no valor de 1,25, além de resíduos com característica de ruído branco, confirmando a qualidade do ajuste.

Como observado, o modelo conseguiu representar a dinâmica da série temporal, reproduzindo a periodicidade e a tendência crescente verificadas na série. Para a previsão no horizonte de doze meses, o modelo SARIMA conseguiu prever as temperaturas médias mensais com baixos valores para os resíduos. 
De modo complementar, destaca-se que a metodologia Box \& Jenkins utilizada mostrou-se adequada para a modelagem de dados climatológicos. Espera-se que tal estudo possa auxiliar no planejamento de diversas atividades, em especial ao agronegócio pujante na região de estudo, considerando-se também para trabalhos futuros estimativas diárias ou de temperaturas mínimas.

\section{REFERÊNCIAS}

AKAIKE, H.. A new look at the statistical model identification. IEEE Transactions on Automatic Control., v.19, n.6, p.716723, 1974.

AKAIKE, H.. A Bayesian analysis of the minimum AIC procedure. Annals of the Institute of Statistical Mathematics, v.30, p.9-14, 1978.

BARBOSA, E. C.; SÁFADI, T.; NASCIMENTO, M.; NASCIMENTO, A. C. C.; SILVA, C. H. O.; MANULI, R. C. Metodologia Box \& Jenkins para previsão de temperatura média mensal da cidade de Bauru (SP). Revista Brasileira de Biometria, São Paulo, v.33, n.1, p.104-117, 2015.

BAYER, D. M.; CASTRO, N. M. R.; BAYER, F. M.. Modelagem e previsão de vazões médias mensais do Rio Potiribu utilizando modelos de séries temporais. Revista Brasileira de Recursos Hídricos, v.17, n.2, p.229-239, 2012. DOI: 10.21168/rbrh.v17n2.p229-239

BAYER, F. M.; SOUZA, A. M.. Wavelets e modelos tradicionais de previsão: um estudo comparativo. Revista Brasileira de Biometria, v.28, n.2, p.40-61, 2010.

BLEIDORN, M. T.; PINTO, W. P.; BRAUN, E. S.; LIMA, G.B.; MONTEBELLER, C. A.. Modelagem e previsão de vazões médias mensais do Rio Jucú, ES, utilizando o modelo SARIMA. Irriga, Botucatu, v.24, n.2, p.320-335, 2019. DOI: https://doi.org/10.15809/irriga.2019v24n2p320-335

BOX, G. E. P.; JENKINS, G. M.; REINSEL, G. C.; LJUNG, G. M.. Time Series Analysis: Forecasting and Control. 5 ed. Wiley, 2016.

CHATFIELD, C.; XING, H.. The analysis of time series: an introduction with R. 7 ed. Boca Raton: CRC Press. 2019

CHECHI, L.; BAYER, F. M.. Modelos univariados de séries temporais para previsão das temperaturas médias mensais de Erechin. Revista Brasileira de Engenharia Agrícola e Ambiental, v.16, p.1321-1329, 2012. DOI: http://dx.doi.org/10.1590/S1415-43662012001200009
COX, D. R.; STUART, A.. Some quick sign test for trend in location and dispersion. Biometrika, v.42, p.80-95, 1955.

IBGE. Instituto Brasileiro de Geografia e Estatística. Portal Cidades. Brasília: IBGE, 2020.

KRISPIN, R.. Hands-on time series analysis with $\mathbf{R}$. Birmingharm: Packt Publishing, 2019.

LJUNG, G. M.; BOX, G. E. P.. On a measure of lack on fit in time series models. Biometrika, v.65, n.2, p.297-303, 1978.

MORETTIN, P. A.; TOLOI, C. M. C.. Análise de séries temporais. 2 ed. São Paulo: Edgard Blucher, 2006.

PEREIRA, A. R.; COSTA, A. S.; OLIVEIRA, V. G.; BORGES, P. F.; FILHO, A. I.. Análise do comportamento das médias anuais da precipitação pluvial e temperatura da cidade de Areia, Paraíba. Gaia Scientia, v.9, n.1, p.67-73, 2015.

R CORE TEAM. R: A language and environment for statistica computing. Vienna: R Foundation for Statistical Computing, 2017.

SAID, S. E.; DICKEY, D. A.. Testing for Unit Roots in Autoregressive-Moving Average Models of Unknown Order. Biometrika, v.71, p.599-607, 1984.

SHAPIRO, S. S.; WILK, M. B.. An analysis of variance test for normality (complete samples). Biometrika, v.52, p.591-611, 1965.

SILVA, M. I. S.; GUIMARÃES, E. C.; TAVARES, M.. Previsão da temperatura média mensal de Uberlândia, MG, com modelos de séries temporais. Revista Brasileira de Engenharia Agrícola e Ambiental, v.12, n.5, p.480-485. 2008.

WERNER, L.; BISOGNIN, C.; MENEGOTTO, L.. Previsão da umidade relativa do ar diária de Porto Alegre. Cadernos do IME, v.43, p.39-59, 2017. DOI: http://doi.org/10.12957/cadest.2017.31211

A CBPC - Companhia Brasileira de Produção Científica (CNPJ: 11.221.422/0001-03) detém os direitos materiais desta publicação. Os direitos referem-se à publicação do trabalho em qualquer parte do mundo, incluindo os direitos às renovações, expansões e disseminações da contribuicão, bem como outros direitos subsidiários. Todos os trabalhos publicados eletronicamente poderão posteriormente ser publicados em coletâneas impressas sob coordenação da Sustenere Publishing, da Companhia Brasileira de Produção Científica e seus parceiros autorizados. Os (as) autores (as) preservam os direitos autorais, mas não têm permissão para a publicação da contribuição em outro meio, impresso ou digital, em português ou em tradução. 